\title{
Fingerprint Matching Based on Octantal Nearest-Neighbor Structure and Core Points
}

\author{
Li-min Yang $^{1}$, Jie Yang ${ }^{1}$, and Hong-tao $\mathrm{Wu}^{2}$ \\ ${ }^{1}$ Institute of Image Processing and Pattern Recognition, Shanghai JiaoTong University (SJTU), \\ Shanghai, 200240, P.R. China \\ ${ }^{2}$ School of Computer Science and Software, Hebei University of Technology, \\ Tianjin, 300130, P.R. China \\ \{ylm, jieyang\}@sjtu.edu.cn, wht_121@163.com
}

\begin{abstract}
In this paper, we propose a novel Octantal Nearest-neighbor Structure and core points based fingerprint matching scheme. A novel fingerprint feature named the octantal nearest-neighbor structure (ONNS) is defined. Based on the ONNS, the minutiae pairing algorithm is conducted to find the corresponding minutiae pairs, and a novel algorithm is developed to evaluate the translational and rotational parameters between the input and the template fingerprints. Core point based orientation pairing is performed thereafter. Matching score is calculated. Experimental results on the FVC2004 fingerprint databases show the good performance of the proposed algorithm.
\end{abstract}

Keywords: Core point; Corresponding minutia pair; Fingerprint alignment; Fingerprint matching.

\section{Introduction}

Most of the fingerprint matching approaches introduced in the last four decades are minutia-based [1]. However, minutiae matching faces a series of challenges such as the location and orientation errors of detected minutiae, as well as the presence of spurious minutiae and the absence of genuine minutiae. One of the most difficult problems to overcome is the nonlinear distortion introduced when the threedimensional surface of the finger is mapped onto a two-dimensional surface by the fingerprint acquisition technique.

Many researchers have tried to address the problem of nonlinear distortion to make the minutiae matching method more robust [2-10]. In [2], a minutiae matching method based on both the local and global structures is proposed. The local structure of a central minutia is used to find the correspondence of two minutiae sets. The best matching pair is then selected and used for registering the input and the template fingerprints. The global structure is used to enforce the result of local matching and a final score is computed to determine the uniqueness of a fingerprint. Similarly in [5], the defined local topological structure (LTS) includes the minutiae circled around the central minutia within $r$ radius.

Different from the above two methods, a fingerprint feature named the adjacent feature vector (AFV) is proposed for fingerprint matching in [3]. For a central 
minutia, its four adjacent points with the same distance 'ADis' to the central minutia are selected to compute four adjacent relative orientations and six ridge counts, which are rotation and translation invariant. It is worth to notice that ADis is also a constant. If $A D i s$ is small, the orientation field in a small region becomes less discriminable. Even two different small regions may have the similar AFV. If ADis is large, the nonlinear distortion can severely impact the distance between two AFVs. In such case, the same central minutia in different impressions may not be considered as a genuine minutia pair.

In this paper, we propose a novel fingerprint matching scheme, which is based on the octantal nearest-neighbor structure (ONNS) and core points. For each central minutia, the nearest neighboring minutia (NNM) in each octant is extracted to construct the local feature structure. Based on the ONNS, the minutiae pairing algorithm is conducted to find the corresponding minutiae pairs. To reduce alignment error, a novel algorithm is developed to evaluate the translational and rotational parameters between the input and template fingerprints. The core points are used to register the orientation fields of input and template fingerprint images. The final matching score measures both the similarity level of two sets of minutiae and two orientation fields. It helps to decrease both the false acceptance rate (FAR) and the false reject rate (FRR). This paper is organized as follows. Section 2 describes the proposed ONNS in detail. Section 3 presents the novel fingerprint matching algorithm. The experimental results are reported in Section 4. Section 5 concludes this paper.

\section{Octantal Nearest-Neighbor Structure}

Let $\left\{M_{i}=\left(x_{i}, y_{i}, \theta_{i}, q_{i}\right)\right\}_{i=1,2, \ldots, N}$ denote the extracted minutiae from the given fingerprint, where $\left(x_{i}, y_{i}\right)$ is its coordinate, $\theta_{i}$ is the local ridge orientation, and the quality $q_{i}$ has the range [1,100]: $q=100$ implies the neighborhood of the minutia has higher possible quality while $q=1$ represents the lowest possible quality. Although the ridge orientation has the range $\left[0,180^{\circ}\right)$, the minutia can be directed into the range $\left[0,360^{\circ}\right)$ to increase its discrimination [2].

To simplify the description of the ONNS, the difference between two angles $\theta_{i}$ and $\theta_{k}$ is defined by a function $d\left(\theta_{i}, \theta_{k}\right)$ :

$$
d\left(\theta_{i}, \theta_{k}\right)=\left\{\begin{array}{lrc}
\theta_{i}-\theta_{k}, \quad \text { if } & -180^{\circ} \leq \theta_{i}-\theta_{k}<180^{\circ} \\
360^{\circ}-\theta_{i}+\theta_{k}, & \text { if } & \theta_{i}-\theta_{k} \geq 180^{\circ} \\
360^{\circ}+\theta_{i}-\theta_{k}, & \text { if } & \theta_{i}-\theta_{k}<-180^{\circ}
\end{array}\right.
$$

For a minutia $M_{i}=\left(x_{i}, y_{i}, \theta_{i}, q_{i}\right)$, its octant is defined as follows:

$$
\left\{\left[\theta_{i}+(j-1) \times 45^{\circ}, \theta_{i}+j \times 45^{\circ}\right)\right\}_{j=1}^{8}
$$


Denote $f\left(\theta_{i}, j\right)$ as the $j$ th octant of $M_{i}$. Let $\beta=\theta_{i}+(j-1) \times 45^{\circ}$, then $f\left(\theta_{i}, j\right)=\left[\beta, \beta+45^{\circ}\right)$. Because $\beta$ and $\beta+45^{\circ}$ may be bigger than $360^{\circ}, f\left(\theta_{i}, j\right)$ should be modulated as follows:

$$
f\left(\theta_{i}, j\right)=\left\{\begin{array}{l}
{\left[\beta, \beta+45^{\circ}\right), \quad \text { if } \beta+45^{\circ}<360^{\circ}} \\
{\left[\beta, 360^{\circ}\right) \cup\left[0^{\circ}, \beta+45^{\circ}-360^{\circ}\right), \quad \text { if } \beta<360^{\circ} \leq \beta+45^{\circ}} \\
{\left[\beta-360^{\circ}, \beta+45^{\circ}-360^{\circ}\right), \quad \text { if } \quad \beta \geq 360^{\circ}}
\end{array}\right.
$$

For the central minutia $M_{i}$, its $j$ th NNM “ $P_{i j}$ " within $f\left(\theta_{i}, j\right)$ is defined as follows:

$$
P_{i j}=\min _{M_{k} \in f\left(\theta_{i}, j\right)} D_{M_{i} M_{k}}, j=1,2, \cdots, 8
$$

where $D_{M_{i} M_{k}}$ denotes the distance between the central minutia $M_{i}$ and minutia $M_{k}$.

The definition of the novel feature structure named the ONNS is given as follows:

$$
F_{M_{i}}=\left\{\left(D_{M_{i} P_{i j}}, C_{M_{i} P_{i j}}, d\left(\theta_{i}, \alpha_{i j}\right), d\left(\theta_{i}, \theta_{j}\right), h\left(q_{i}, q_{j}\right)\right)\right\}_{j=1,2, \ldots, 8}
$$

where $D_{M_{i} P_{i j}}$ denotes the distance between central minutia $M_{i}$ and minutia $P_{i j}, C_{M_{i} P_{i j}}$ denotes the ridge-counts between $M_{i}$ and $P_{i j}, \alpha_{i j}$ denotes the direction of the directed line segment $\overrightarrow{M_{i} P_{i j}}$, and $h\left(q_{i}, q_{j}\right)$ denotes the joint quality between the quality $q_{i}$ of $M_{i}$ and that of $P_{i j}$. Refer to Fig.1 for clarification.

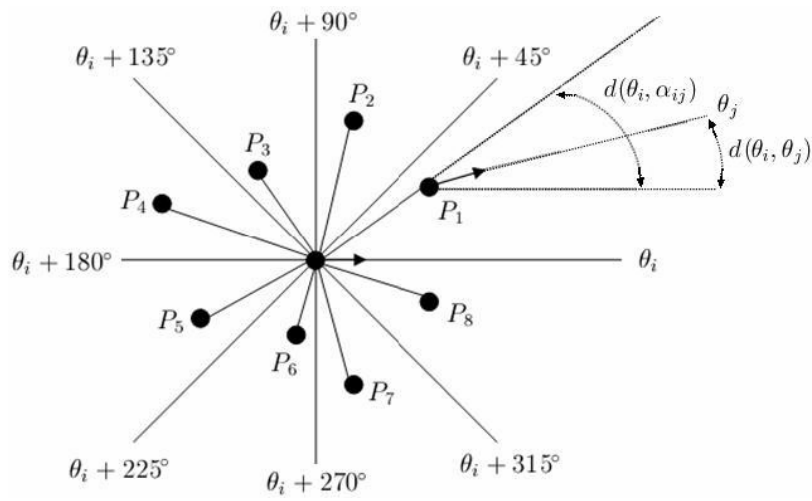

Fig. 1. Illustration of octantal nearest-neighbor structure

The number of ridges between two minutiae and the quality of each minutia should be calculated in the minutiae detection phase. In this work, the joint quality between $q_{i}$ and $q_{j}$ is calculated by $h\left(q_{i}, q_{j}\right)=q_{i} \times q_{j}$. 


\section{Fingerprint Matching}

Using the proposed ONNS, we develop a new fingerprint matching algorithm making use of both fingerprint minutiae and core points.

\subsection{Corresponding Minutiae Pairs Identification}

To identify whether two minutiae are corresponding minutia pair, we need to calculate the similarity level between their ONNSs first:

Suppose $F_{M_{i}}^{I}$ and $F_{M_{j}}^{T}$ are the ONNS of minutia $M_{i}^{I}$ from the input fingerprint and that of minutia $M_{j}^{T}$ from the template fingerprint, respectively. The following algorithm is used to calculate the similarity level $S\left(M_{i}^{I}, M_{j}^{T}\right)$ between $M_{i}^{I}$ and $M_{j}^{T}$.

Step 1: Compute the similarity level $S_{k}\left(M_{i}^{I}, M_{j}^{T}\right)$ at the $k$ th octant between $M_{i}^{I}$ and $M_{j}^{T}$ :

If the $k$ th NNM $P_{i k}^{I}$ and $P_{j k}^{T}$ both exist and the following conditions are satisfied:

$$
\begin{gathered}
D_{i j k}=\mid D_{M_{i}^{I} P_{i k}^{I}}-D_{M_{j}^{T} P_{j k}^{T}}<T_{D} \\
C_{i j k}=\mid C_{M_{i}^{I} P_{i k}^{I}}-C_{M_{j}^{T} P_{j k}^{T}}<T_{C} \\
\gamma_{i j k}=d d\left(d\left(\theta_{i}^{I}, \alpha_{i k}^{I}\right), d\left(\theta_{j}^{T}, \alpha_{j k}^{T}\right)\right) k T_{\theta} \\
\eta_{i j k}=d d\left(d\left(\theta_{i}^{I}, \theta_{k}^{I}\right), d\left(\theta_{j}^{T}, \theta_{k}^{T}\right)\right) k T_{\theta}
\end{gathered}
$$

then the similarity level $S_{k}\left(M_{i}^{I}, M_{j}^{T}\right)$ is calculated as follows:

$$
S_{k}\left(M_{i k}^{I}, M_{j k}^{T}\right)=h\left(h\left(q_{i}^{I}, q_{k}^{I}\right), h\left(q_{j}^{T}, q_{k}^{T}\right)\right)\left(1-\frac{\left(\frac{w_{D} D_{i j k}}{T_{D}}+\frac{w_{C} C_{i j k}}{T_{C}}+\frac{w_{\theta}\left(\gamma_{i j k}+\eta_{i j k}\right)}{T_{\theta}}\right)}{w_{D}+w_{C}+2 w_{\theta}}\right)
$$

If $P_{i k}^{I}$ or $P_{j k}^{T}$ does not exist, or the conditions Equation(5)-(8) are not all satisfied, $S_{k}\left(M_{i}^{I}, M_{j}^{T}\right)$ should be set to zero. The coefficients (i.e., $w_{D} 、 w_{C}$ and $w_{\theta}$ ) specify the weights associated with the corresponding component of the ONNS. The three thresholds(i.e., $T_{D} 、 T_{C}$ and $T_{\theta}$ ) are the size of matching box, which change according to the distance $D_{i j k}$, as described in [5]. For example:

$$
T_{D}=\left\{\begin{array}{l}
T_{D L}, \text { if } \quad D_{i j k}<T_{L} \\
T_{D H}, \text { if } D_{i j k}>T_{H} \\
T_{D L}+\frac{T_{D H}-T_{D L}}{T_{H}-T_{L}}\left(D_{i j k}-T_{L}\right), \text { otherwise }
\end{array}\right.
$$


Here, $T_{L}, T_{H}, T_{D L}$ and $T_{D H}$ are four pre-established parameters.

Step 2: Calculate the similarity level $S\left(M_{i}^{I}, M_{j}^{T}\right)$ between $M_{i}^{I}$ and $M_{j}^{T}$ :

$$
S\left(M_{i}^{I}, M_{j}^{T}\right)=h\left(q_{i}^{I}, q_{j}^{T}\right) \sum_{k=1}^{8} S_{k}\left(M_{i}^{I}, M_{j}^{T}\right)
$$

The similarity level $S\left(M_{i}^{I}, M_{j}^{T}\right)$ describes a matching certainty level of an ONNS pair instead of simply matched or not. $S\left(M_{i}^{I}, M_{j}^{T}\right)=1$ implies a perfect match while $S\left(M_{i}^{I}, M_{j}^{T}\right)=0$ represents a total mismatch. From Fig.2 to Fig.4, we show an example of the proposed ONNS.

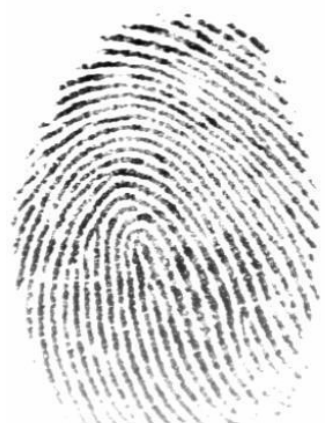

Fig. 2. A fingerprint image named "33 6.tif" from FVC2004 DB1A.

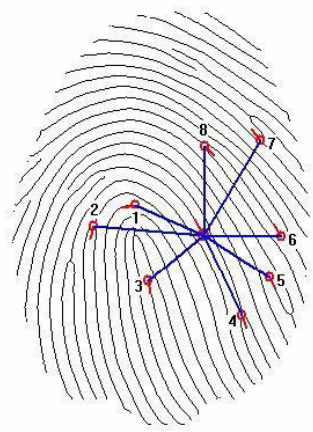

Fig. 4. An example of the ONNS: the NNM of the central minutia in each octant all exists

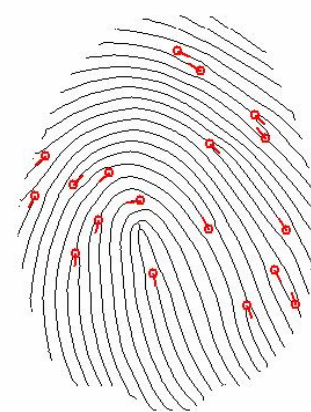

Fig. 3. Extracted minutiae and their directions of the image "33 6.tif"

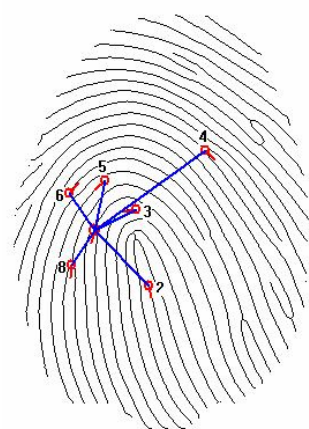

Fig. 5. Another example of the ONNS: there is not a NNM in the 1st and 7th octants of the central minutia

If $S\left(M_{i}^{I}, M_{j}^{T}\right)>T_{s}$ ( $T_{s}$ is a threshold), $M_{i}$ and $M_{k}$ are considered a corresponding minutiae pair. Let $\left\{\left(M_{p_{j}}^{I}, M_{q_{j}}^{T}\right): j=1,2, \ldots, L\right\}(L \leq K)$ denote the detected corresponding minutiae pairs. 


\subsection{Translational and Rotational Parameters Estimation}

After the corresponding minutiae pairs are identified, the global translational and rotational parameters between the input and the template fingerprints can be evaluated as follows:

1) Calculate the mass center of the corresponding minutiae:

$$
\left[\begin{array}{l}
x_{0}^{I} \\
y_{0}^{I}
\end{array}\right]=\frac{1}{L}\left[\begin{array}{c}
\sum_{j=1}^{L} x_{p_{j}}^{I} \\
\sum_{j=1}^{L} y_{p_{j}}^{I}
\end{array}\right],\left[\begin{array}{l}
x_{0}^{T} \\
y_{0}^{T}
\end{array}\right]=\frac{1}{L}\left[\begin{array}{c}
\sum_{j=1}^{L} x_{q_{j}}^{I} \\
\sum_{j=1}^{L} y_{q_{j}}^{I}
\end{array}\right]
$$

2) Compute the translational parameters $\Delta x$ and $\Delta y$ :

$$
\left[\begin{array}{c}
\Delta x \\
\Delta y
\end{array}\right]=\left[\begin{array}{c}
x_{0}^{T}-x_{0}^{I} \\
y_{0}^{T}-y_{0}^{I}
\end{array}\right]
$$

3) Evaluate the rotational parameter $\Delta \theta$ :

$$
\Delta \theta=\max _{\theta} \sum_{\left|d\left(\theta, \theta_{0}\right)\right| \leq \alpha_{0}}\left(\alpha_{0}+1-\left|d\left(\theta, \theta_{0}\right)\right|\right) S_{\theta}
$$

where $\alpha_{0}$ is a parameter(in this work $\alpha_{0}=15^{\circ}$ ), and

$$
\theta_{0}=d\left(\theta_{p_{j}}^{I}, \theta_{q_{j}}^{T}\right), \quad S_{\theta}=\sum_{\theta=\theta_{0}, j=1,2, \ldots, L} S\left(M_{p_{j}}^{I}, M_{q_{j}}^{T}\right)
$$

After obtaining the translational and rotational parameters $(\Delta x, \Delta y, \Delta \theta)$, the new location $\left(x_{i}^{I^{\prime}}, y_{i}^{I^{\prime}}\right)$ for minutia $M_{i}^{I}, i=1,2, \cdots, K_{0}$, can be calculated as follows:

$$
\left[\begin{array}{c}
x_{i}^{I^{\prime}} \\
y_{i}^{I^{\prime}}
\end{array}\right]=\left[\begin{array}{cc}
\cos (\Delta \theta) & \sin (\Delta \theta) \\
-\sin (\Delta \theta) & \cos (\Delta \theta)
\end{array}\right]\left[\begin{array}{c}
x_{i}^{I}-x_{0}^{I} \\
y_{i}^{I}-y_{0}^{I}
\end{array}\right]+\left[\begin{array}{c}
x_{i}^{I}+\Delta x \\
y_{i}^{I}+\Delta y
\end{array}\right]
$$

The direction of each minutia of the input fingerprint is also aligned:

$$
\theta_{i}^{I^{\prime}}=\theta_{i}^{I}+\Delta \theta
$$

Let $M_{i}^{I^{\prime}}=\left\{x_{i}^{I^{\prime}}, y_{i}^{I^{\prime}}, \theta_{i}^{I^{\prime}}, q_{i}^{I^{\prime}}\right\}$ denote the new minutia of the input fingerprint after transformation with the estimated translation and rotation parameters.

\subsection{ONNS Based Pairing}

For the transformed minutiae set $\left\{M_{i}^{I^{\prime}}\right\}_{i=1,2, \cdots, K_{0}}$, we re-compute the ONNS of each minutia. Using the improved algorithm described above, we find the corresponding 
minutiae pairs between the transformed minutiae set $\left\{M_{i}^{I^{\prime}}\right\}_{i=1,2, \cdots, K_{0}}$ and the originally extracted minutiae set $\left\{M_{j}^{T}\right\}_{j=1,2, \cdots, K_{1}} \cdot\left\{M_{c_{k}}^{I^{\prime}}, M_{c_{k}}^{T}\right\}_{k=1,2, \ldots, K^{\prime}}, \quad K^{\prime} \leq \min \left(K_{0}, K_{1}\right)$, denote the corresponding minutiae pairs.

\subsection{Core Point Based Orientation Pairing}

A core is defined as a point in the orientation field where the orientation in a small local neighborhood around the point presents semi-circular tendency. It is a salient feature in a fingerprint image. In this work, the Poincare index introduced by [13] is used to extract core points. Fig. 6 shows example of an extracted core point.

Notice that there may be one or two core point(s) in one fingerprint image, we discuss the one core point cases first.

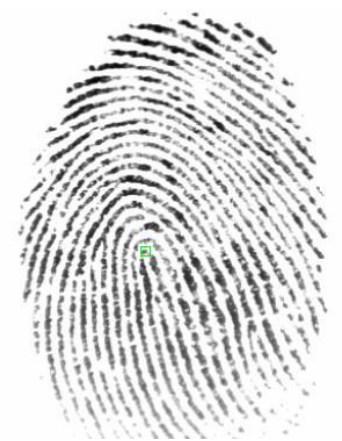

Fig. 6. An extracted core point

Assume $\operatorname{Core}^{I}\left(x^{I}, y^{I}, \theta^{I}\right)$ and $\operatorname{Core}^{T}\left(x^{T}, y^{T}, \theta^{T}\right)$ are core points from input fingerprint and template fingerprint, respectively. Calculate the translational and rotational parameters $\left(\Delta x_{c}, \Delta y_{c}, \Delta \theta_{c}\right)$ as follows:

$$
\left[\begin{array}{l}
\Delta x_{c} \\
\Delta y_{c} \\
\Delta \theta_{c}
\end{array}\right]=\left[\begin{array}{c}
x^{T}-x^{I} \\
y^{T}-y^{I} \\
\theta^{T}-\theta^{I}
\end{array}\right]
$$

By translating and rotating the input fingerprint image according to parameter $\left(\Delta x_{c}, \Delta y_{c}, \Delta \theta_{c}\right)$, a new transformed input fingerprint image is obtained.

Divide the transformed input fingerprint image and the template fingerprint image into a number of sub-blocks, then calculate the orientation of each sub-block[11]. Let $\left(B_{i}^{I^{\prime}}, B_{i}^{T}\right)$ denote the corresponding orientation block pair, block $B_{i}^{I^{\prime}}$ from the transformed input fingerprint, block $B_{i}^{T}$ from the template fingerprint, respectively, $i=1,2, \cdots, N_{B}, N_{B}$ is the number of blocks in one image. The similarity level $S\left(B_{i}^{I^{\prime}}, B_{i}^{T}\right)$ of the two blocks $B_{i}^{I^{\prime}}$ and $B_{i}^{T}$ is calculated as follows: 


$$
S\left(B_{i}^{I^{\prime}}, B_{i}^{T}\right)=\left\{\begin{array}{cc}
\frac{T_{B}-\left|O\left(B_{i}^{I^{\prime}}\right)-O\left(B_{i}^{T}\right)\right|}{T_{B}} & \text { if } \\
0 & \left|O\left(B_{i}^{I^{\prime}}\right)-O\left(B_{i}^{T}\right)\right|<T_{B}, \\
\text { otherwise. }
\end{array}\right.
$$

where $T_{B}$ is a threshold, and $O(\bullet)$ denotes the orientation of a block.

If there are two core points in the input image or template image, or two core points in both fingerprint images, each core point in one image would pair with all the core point(s) in the other image to get a set of transform parameters. Repeat the pairing procedure described above. The core point pair with the maximal summatory similarity level is considered as the corresponding core point pair.

\subsection{Matching Score Computation}

The final matching score $M s$ between input and template fingerprints can be determined by minutia matching score $M_{m}$ and core point based matching score $M_{c}$. The minutia matching score $M_{m}$ can be calculated according to the following equation:

$$
M_{m}=\sum_{k=1}^{K^{\prime}} S\left(M_{c_{k}}^{I^{\prime}}, M_{c_{k}}^{T}\right)
$$

where $\left(M_{c_{k}}^{I^{\prime}}, M_{c_{k}}^{T}\right)$ is the corresponding minutiae pair, one from the transformed input fingerprint and another from the original template fingerprint, respectively.

The core point based matching score $M_{c}$ is defined by

$$
M_{c}=\sum_{i=1}^{N_{B}} S\left(B_{i}^{I^{\prime}}, B_{i}^{T}\right)
$$

where $\left(B_{i}^{I^{\prime}}, B_{i}^{T}\right)$ is the corresponding orientation pair, one from the transformed input fingerprint and another from the original template fingerprint, respectively.

If there are no core points or over two core points detected in input or template fingerprint due to poor quality or other reasons, $M_{c}$ should be set to zero.

The final matching score $M s$ is computed as follows:

$$
M s=\alpha_{m} M_{m}+\alpha_{c} M_{c}
$$

where $\alpha_{m}$ and $\alpha_{c}$ are weights, and $\alpha_{m}=1-\alpha_{c}$ (in this work, if $M_{c}=0$, then $\alpha_{c}=0$ otherwise $\alpha_{c}=0.4$ ).

\section{Results}

The proposed scheme has been evaluated by applying to DB1A and DB3A of FVC2004, because the distortion between some fingerprints from the same finger in these two databases is large. The fingerprints of DB1A are acquired through 
CrossMatch "V300" optical sensor, and each fingerprint is with $640 \times 480$ pixels and 500 dpi. The fingerprints of DB3A are acquired through thermal sweeping sensor "FingerChip FCD4B14CB" by Atmel, and each fingerprint is with $300 \times 480$ pixels and 512 dpi. Both databases contain 800 fingerprints captured from 100 different fingers, eight impressions for each finger.

Table 1. Comparison of the proposed scheme with algorithm P026 on FVC2004 DB1A

\begin{tabular}{cccccc}
\hline Algorithm & EER & $\begin{array}{c}\text { FMR } \\
100\end{array}$ & $\begin{array}{c}\text { FMR } \\
1000\end{array}$ & $\begin{array}{c}\text { Average } \\
\text { enroll time }\end{array}$ & $\begin{array}{c}\text { Average } \\
\text { match time }\end{array}$ \\
\hline Our scheme & $5.36 \%$ & $7.44 \%$ & $12.40 \%$ & $2.11 \mathrm{~s}$ & $2.32 \mathrm{~s}$ \\
P026 & $5.54 \%$ & $9.25 \%$ & $19.11 \%$ & $2.60 \mathrm{~s}$ & $3.56 \mathrm{~s}$ \\
\hline
\end{tabular}

Table 2. Comparison of the proposed scheme with algorithm P004 on FVC2004 DB3A

\begin{tabular}{cccccc}
\hline Algorithm & EER & $\begin{array}{c}\text { FMR } \\
100\end{array}$ & $\begin{array}{c}\text { FMR } \\
1000\end{array}$ & $\begin{array}{c}\text { Average } \\
\text { enroll time }\end{array}$ & $\begin{array}{c}\text { Average } \\
\text { match time }\end{array}$ \\
\hline Our scheme & $1.86 \%$ & $2.25 \%$ & $5.23 \%$ & $0.84 \mathrm{~s}$ & $1.02 \mathrm{~s}$ \\
P004 & $1.89 \%$ & $2.61 \%$ & $7.14 \%$ & $0.76 \mathrm{~s}$ & $0.80 \mathrm{~s}$ \\
\hline
\end{tabular}

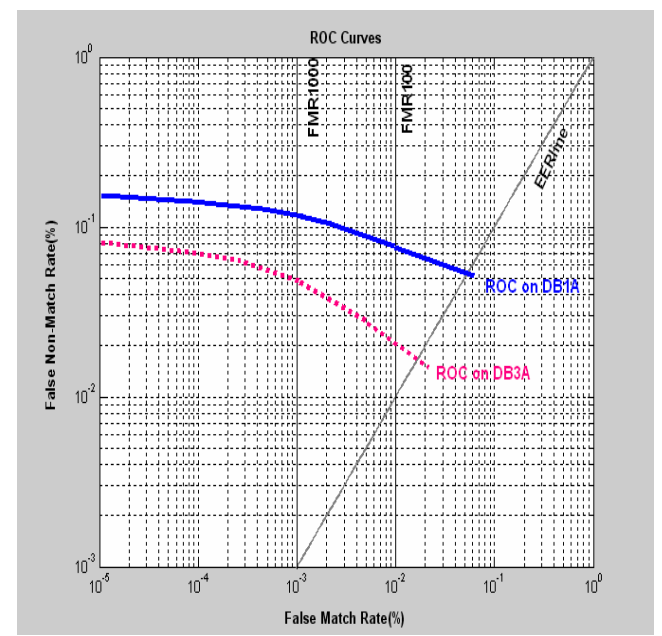

Fig. 7. The ROC curves obtained by the proposed scheme on FVC2004 DB1A and DB3A

In Table 1 and Table 2, We compare the results of the proposed scheme on DB1A with that of the algorithm called "P026", and compare the results of the proposed scheme on DB3A with that of the algorithm called "P004", which have been participated in FVC2004, and ranked the ninth and the sixth places respectively. According to the ranking rule in terms of EER in FVC2004, the proposed scheme is better than the two algorithms, which means a good performance both for the DB1a and DB3a. In FVC2004, the maximum time cost for each enrollment and each 
matching is 10 seconds and 5 seconds, respectively[12]. Table 1 and Table 2 show that the time cost of the proposed scheme satisfies the conditions of the open category on FVC2004. The receiver operating characteristic (ROC) curves obtained by the proposed scheme on FVC2004 DB1A and DB3A are illustrated in Fig.7.

\section{Conclusions}

In this paper, we propose an ONNS and core point based fingerprint matching scheme. A novel feature structure ONNS is defined, which is translation and rotation invariant. Based on ONNS and core points, fingerprint matching is performed. Because both minutiae information and core points information are used in this work, it is more robust than minutiae-based methods. The usefulness of our proposed approach is confirmed in the experiments conducted, which show good performance. However, there are still some difficulties to be overcome in the future, such as how to locate minutiae and core points in poor quality fingerprints more reliably, and how to solve the problems introduced by nonlinear distortion. Those would be our future directions.

\section{References}

1. Davide Maltoni, Dario Maio, Anil K. Jain, and Salil Prabhakar: Handbook of Fingerprint Recognition. Springer-Verlag, New-York (2003)

2. Xudong Jiang, Wei-Yun Yau: Fingerprint Minutiae Matching Based on the Local and Global Structures. ICPR 2000, 2, (2000)1042-1045

3. Xifeng Tong, Jianhua Huang, Xianglong Tang, Daming Shi: Fingerprint Minutiae Matching Using the Adjacent Feature Vector. Pattern Recognition Letters, 26(9), (2004)1337-1345

4. En Zhu, Jiangping Yin, Guomin Zhang: Fingerprint Matching based on Global Alignment of Multiple Reference Minutiae. Pattern Recognition, 38(10), (2005)1685-1694

5. Xinjian Chen, Jie Tian, Xin Yang: A New Algorithm for Distorted Fingerprint Matching Based on Normalized Fuzzy Similarity Measure. IEEE translations on Image Processing, 15(3), (2006)767-776

6. Jin Qi, Yangsheng Wang: A Robust Fingerprint Matching Method. Pattern Recognition, 38(10), (2005)1665-1671

7. Arun Ross, Sarat C. Dass, Anil K. Jain: Fingerprint Warping Using Ridge Curve Correspondences. IEEE transactions on Pattern Analysis and Machine Intelligence, 28(1), (2006)19-30

8. Asker M. Bazen, Sabih H. Gerez: Fingerprint Matching by Thin-plate Spline Modelling of Elastic Deformations. Pattern Recognition, 36(8), (2003)1859-1867

9. Arun Ross, Sarat C. Dass, Anil K. Jain: A Deformable Model for Fingerprint Matching. Pattern Recognition, 38(1), (2005)95-103

10. Neil Yager, Adnan Admin: Nonparametric Fingerprint Deformation Modelling. CAIP 2005, LNCS 3691, (2005)73-80

11. Anil K. Jain, Lin Hong, and Ruud Bolle: On-Line Fingerprint Verification. IEEE Transactions on Pattern Analysis and Machine Intelligence, 19(4), 302-314 (1997)

12. Biometric Systems Lab., Pattern Recognition and Image Processing Lab., Biometric Test Center., [Online] Available: http://bias.csr.unibo.it/fvc2004/

13. Kawagoe, M., Tojo, A.: Fingerprint pattern classification. Pattern Recognition 17 (3), (1984)295-303 